\title{
An Experience of Otorhinolaryngologists as Frontline Worker with Novel Coronavirus: A Qualitative Analysis
}

\author{
Rajwant Kaur ${ }^{1}$ (D) Amanjot Kaur $^{2} \cdot$ Pawan $_{K_{u m a r}^{3}}$
}

Received: 17 December 2020/ Accepted: 4 January 2021 / Published online: 16 January 2021

(C) Association of Otolaryngologists of India 2021

\begin{abstract}
During this COVID pandemic healthcare worker systems were overwhelmed. Doctors especially otorhinolaryngologists in addition to doing their specialty duty were also recruited to provide care to COVID-19 patients. To our knowledge, no studies about their experiences regarding COVID-19 have been published. Present study aimed to describe the experiences of Otorhinolaryngologist during this pandemic. This is a qualitative study using an empirical phenomenological approach. 30 ENT doctors were recruited from government and private sector. They participated in semi-structured, in-depth interviews by telephone in a period of one month. Interviews were recorded by consent and data was analyzed. After analysis four themes were revealed namely; 'nature of duty during Covid-19 pandemic', 'modification made to adjust the duty', 'conflict between professional duty and family responsibilities', and 'fear of pandemic ill effect on their health'. The extreme work and fatigue drained ours ENT doctor's physically as well as emotionally. But our doctors showed their resilience and the spirit of professional dedication to overcome difficulties. Comprehensive support should be provided to safeguard the wellbeing of healthcare providers mainly doctors. In the meantime timely as well as intensive training for all healthcare worker is highly recommended to promote preparedness and improve efficacy during the pandemic.
\end{abstract}

Rajwant Kaur

rajwin000@gmail.com

1 Department of ENT, Govt Rajindra Hospital, Flat E-13, New Lal Bagh Colony, Patiala, Punjab 147001, India

2 ENT, Civil Hospital, Sangrur, India

3 Orthopaedics, AP Healthcare, Patiala, India
Keywords Otorhinolaryngologists - Coronavirus · Isolation ward $\cdot$ Emergency $\cdot$ Telehealth

\section{Introduction}

Coronavirus disease 2019 (COVID-19), caused by severe acute respiratory syndrome coronavirus 2 (SARS-CoV-2), has been declared pandemic by the World Health Organization on March 2020. Millions of people around world are under the effect of this pandemic but the health care workers are completely invested in form of treating and caring the infected peoples. So our health care workers are directly or indirectly subjected to high risk of contracting this new virus.

Presently our front-line workers especially our doctors are succumbed by Covid-19 infections. Unfortunately many of them lost their lives while treating COVID-19 patients. All specialties doctors are equally affected. But as this new disease is transmitted through aerosols so our otorhinolaryngologists are more in fear of contacting this disease. A recent study conducted in Wuhan hospital by Ren $\mathbf{J}$ et al. during early outbreak of disease, a large number of healthcare workers were firstly diagnosed for Covid-19 infection in the Department of Otorhinolaryngology only [1]. Most of them were involved in the care of ENT patients who was later on diagnosed with COVID-19. As ENT people are involved in both aerosol generating diagnostic and emergency surgical procedures. During local examination as well as management of patients ENT people are in close contact with the airway and its secretions and aerosol generation. Viral loads high in the nasal and oropharyngeal mucosa, which is the main route of severe acute respiratory syndrome (SARS) transmission $[2,3]$. As most of the patients are asymptomatic during 
their visits. Similarly maximum corona symptoms like nasal stuffiness, sore throat, loss of smell and taste are dealt under ENT specialty. At the same time emergency situation like epistaxis, for respiratory failure or any malignant growth of the upper airway where tracheostomy is the only lifesaving procedure which maximally generating aerosols especially during inadvertent sudden bout of cough. The risk is even more in asymptomatic and not previously tested for Covid due to emergency, this increase the susceptibility for contracting the disease. It was evident in similar studies from China, Iran, Italy, and United States $[4,5]$.

Therefore, the aims of present study was to know the actual experience of otorhinolaryngologists working in Covid-19, through semi structured interviews, discussing mainly the role of otorhinolaryngologists in diagnosis, care of isolated and infected patients and as well as their treatment. Modification they did to protect themselves and how challenging the duty was for them, and by what means it effected their well-being. Accordingly four themes were revealed namely; 'nature of duty during Covid-19 pandemic', 'modification made to adjust the duty', 'conflict between professional duty and family responsibilities', and 'fear of pandemic ill effect on their health'. Data was analyzed using phenomenological methods. By the study we able to elucidate the challenges our otorhinolaryngologists facing during pandemic, how they prepared themselves, and what was the new the effect of this pandemic and the preparedness on their health both physical, mental and social well-being.

\section{Material and Method}

The present study was conducted in one month period. This is a qualitative region based study on 30 otorhinolaryngologists working in Covid-19. Interviews was conducted telephonically and verbal consent for participation was taken before hand with assurance of confidentiality. Phenomenological method was used for data analysis. Participants were contacted through telephone in their free time 30 to $40 \mathrm{~min}$ interview was conducted and audio recorded with their permission. Emotional support was provided whenever it was required and the participant was free to withdraw the consent at any time during the interview process. Interviewed question was mainly based what was the nature of their duty during Covid-19 pandemic, what kind of modification they made to adjust their duty, any type of conflict between professional duty and family responsibilities, and the fear of pandemic ill effect on their health. In addition to this many supplementary questions were asked, self-reported demographic characteristic that included age, sex, and marital status, no. of kids, living with or away from family. Semi structured interview was done and open-ended follow up questions were asked to obtained detailed descriptions, like "did you feel any difference providing your services during this pandemic and non-pandemic time."; "what challenge did you encounter during duty hours?"; many probing questions like "please give detailed description."

"Did you feel any difference between providing their health service during this pandemic and about your own specialties?"

"What challenges did you faced during your working hours."

"What modification did you made to adjust or adapt these changes?"

"What you are doing to protect yourself from undue risk of harm?"

"How did you respond toward your duty or to your family?"

"How are you feeling about yours duty for society?"

"What kind of external or internal support you received and what you are expecting?"

Data collection done concurrently with data analysis. Audio recording was reviewed again and again for data analysis and interviewer accuracy. After analyzing the data, choosing the quotation to be highlighted, the result was concluded.

\section{Data Analysis}

The collected data analyzed by repeated listening to understand the actual meaning of their talked. For analyzing the data audio recording was reviewed several times for understanding the meaning and for identification of significant phrases. These finding were then compared and discussed by the authors to formulate and validate meaning into four main themes. These themes was clustered and further categorized. To establish the transferability variations in participant characteristic was thoroughly observed and interesting quotations was collected after deep analysis of their interviews. Our otorhinolaryngologists are working on frontline as well doing emergency and OPD duties.

\section{Results}

The present study enrolled 37 otorhinolaryngologists selected randomly for participation. Only 30 were interested to participate rest 7 refused because of the tiredness and overwork. All 30 doctors was working in both private and government sectors, and are interested to participate and given their verbal consent for that, however their 
privacy and confidently was maintained throughout the study. 27 doctors were working in government sector and rest 3 was doing their private practice. The mean age the participant was 41.13 female and 17 male doctors was there and it was region based study. 28 are married, 26 having kids, 23 living with family and 7 away. The interview took approximately 30-40 min. Our otorhinolaryngologists are working on frontline as well doing emergency and OPD duties. All the 27 doctors of public sector working on frontline that include taking swab sample and doing isolation duty. In addition to this during their nonCovid duties day they are during routine OPD and emergency duties. Rest 3 were in private sectors and involved in OPD and elective procedure. Possibility of contracting the infection from asymptomatic OPD and presenting with emergency was very high [6].

ENT doctors who are working in OPD, have to screen the patients thoroughly for Covid sign and symptoms. In case of suspicion PCR test for Covid-19 and chest X-ray done, simultaneously patient volume was controlled to decrease the risk of cross-infection. To decrease the risk of transmission during clinical procedures following precaution were used. For oral and nasal cavity examination N99 masks with safety goggles and gloves and if possible shield or disposable fluid resistant gown was used. Endoscopic examination are avoided unnecessarily as high chances of aerosols generation but when laryngopharyngeal or nasal endoscopy is must, by using appropriate PPEs with adequate local anesthesia in less possible time it was completed.

D: As being an ENT doctors we have first point of contact with the patient of flu like symptoms in OPD, during that contact it is not known whether she or he is positive.

D: Every day I see around 60-70 OPD patients and I don't know how many of them are infected with coronavirus. The symptoms of URI and COVID are so similar that it is very difficult to differentiate only on clinical grounds.

D: Nowadays, we are not able to examine the patients the way we used to. Human touch was very common as patients without this never reassured, and for our specialty it is must. But thing are changed we need to diagnose with limited contact, so we need to be more consoling.

D: In OPD patients are coming we are treating and examining them without knowing their Covid status, later some of them turned positive. So some fast testing facilities are needed to avoid unnecessary exposure."

D: we habitually wear masks and are constantly washing our hands after examining every patient. Unless we ourselves are convinced that these precautions will work.
D: social distancing is not at all possible in government as well as private clinics. As most of them are small and congested. Patients with fever and URI have to sit and wait along with other patients so simple masks and gloves are not sufficient to enough to correct other patients as well as doctors. So triaging patients into fever and non-fever categories need to done.

D: In OPDs, we get patients and treat them but later some of them turn out to be Covid positive. Government should ramp up the testing facilities, so that we can get results fast.

For the ENT elective surgery has been postponed according to the guidelines. Due to the preference of virus for airway mucosa and also for middle ear, there is high risk of contamination of ear surgeries [7-10]. But emergency like epistaxis, tracheostomy, Ludwig like abscess drainage without airway compromise, non-life threatening head and neck lacerated wound stitching, other inflammatory and neoplastic diseases foreign bodies of esophagus, EAC, complete PPE is used. It comprised PAPR, face shield, double pair of gloves, proper local anesthesia to avoid unnecessary generat-ion of aerosols during coughing and sneezing. All the procedures are done by senior and expert people to minimize the risk of exposure. In addition to this number of staff is reduced to minimum.

D: According to the government guidelines, fluid resistant FFP3/N95 masks, fluid-resistant and disposable gloves, glasses or face shields are used by us to examine the patient in OPD. Double gloving recommended during surgery.

D: Our senior people started doing emergency procedure, junior residents are withdrawn to avoid exposure to them as they are under training.

D: we are trying our best to protect our self during emergency surgeries. Tracheostomy is most urgent and frequent ENT surgery. It can't be delayed at any cost. So every emergency tracheostomy patients are considered as positive and with complete PPEs measure procedure used to done in separate OT. We are using cuffed and non-fenestrated tracheostomy tube to avoid virus from aerosolizing. After that OT was properly disinfected. Negative pressure or closed suction are used by us to prevent aerosol exposure.

Otorhinolaryngologists working on frontline were doing Covid-19 isolation duties. As treating the sick patients is totally different from their specialties, find themselves in an uncertain position; as they are physicians. No doubt whole world is facing this deadly disease about which much remains unknown, so it seem that they are also trainees, and there is a high probability of it transmissibility as well as pathogenicity. Most of the ENT doctors have no 
experience of working in an intensive care unit or dealing with critically ill patients who requires mechanical ventilation. That added additional mental stress to learn and master new technical procedures in a short time. Doctors expressed that working with PPE for long hours was a major physical and professional challenge.

D: Now I am dealing with the patients who are not in my specialty. No doubt we are working on national guideline but still no effective antiviral medicine for this new disease.

D: I have to work with the medical team from medicine departments in isolation ward. As with ENT knowledge I feel it very stressful to find ways to make the whole process as smooth as possible and ensure the quality of care.

In this tough time doctors are surrounded by positive vibes that motivates doctors to keep working under such strenuous conditions.

D: we keep thinking of innovations.

D: Every day in the hospital, we have training sessions for health care workers, especially the nurses, ward boys, and technicians. They have to be educated about wearing masks and gloves all the time because they are the first ones to attend a patient.

D: the most satisfying part of our profession is that we are in position to make a difference. We must embrace it. D: I talk to the patients, calm them and advise that they should take care of themselves, clear their mind and search for the light amid this darkness.

D: our daily routine has changed, and this new routine has quickly become the norm. I realize that treating patients with Covid-19 is very hard, each one with their own unique needs.

D: we doctors have handled many a disaster, but this is a different kind of crisis. The fear of getting infected is exacting an emotional and mental toll on us. No doubt it's a war and we are soldiers. But we don't want to become martyrs. I trying to be strong and healthy for the fight.

D: I used to listen the devotional chants during the drive to hospital every morning to mentally prepare for the rest of the day.

D: sometime after dinner I love to paint as back to back work at the hospital really tired me. Painting pleased me, relaxed me.

D: I do callisthenics and mostly weight-free workout for about $1-2 \mathrm{~h}$ a day but never to the point of exhaustion. It helps me re-charge my battery.

D: During the duty when our patient becomes COVID negative and becomes fine, it really lifts our spirits and motivates us to do better work and serve our patients and our nation when it is needed the most,"

D: We suppose to maintain social distance when we are outside. So I completely avoid going out only in extreme emergency I stepped out. You can imagine how lonely and isolated we are. But for doctors like us, service comes first, even when you have to wear protective gear for six hours.

D: People often talk about health care workers as soldiers or troops "on the front lines." I think this is an imperfect analogy. While there is no doubt that we are putting our lives on the line every day, our intention is not to make war on this virus, but to heal people.

Doctors feeling overwhelmed by the magnitude of the work. But there are some story of recovery that has inspired them to keep going. One such story by one of our doctors really inspiring.

D: We were paged to the ER for a patient in respiratory distress. I was astonished to find a person in their early $30 \mathrm{~s}$, with no known past medical history, labouring to breathe. This patient was a year younger than I am. We put ET tube and the patient remained in the ICU for two weeks. One day in mid-April, I was overjoyed to find that the patient had been removed from the ventilator and had left the ICU. This young person has a chance at a normal. I often remind myself about this patient after a particularly difficult or stressful shift.

For Otorhinolaryngologists working as frontline, the pandemic has been relentless. It has led to anxiety and fear as the risk of exposure is double-edged, at the workplace as well as outside the hospital premises.

D: I am extremely worried for my family, thinking about the risk of taking care of our families member is the major part of our lives now.

D2: it is difficult to diagnose asymptomatic staff member, as virus is very smart infect very easily. So are staff member provided with the option of using hospital accommodation, especially for those who are working late hours and those who may not want to go back to their families.

Doctors are always under the fear of exposing their loved one to COVID-19. So there are trying to make distance from them and forced to be emotionally alone.

D: I am sharing common area with my family, so I tried hard not to pass any potential infection on by touching anything. My apparels are washed immediately as soon as I am home.

D: I didn't get close to my pregnant wife in a fear to infect her. 
D: Last week, a doctor working with me started showing COVID symptoms. He tested positive and was admitted to the same corona ward where other patients are being treated. After my colleague tested positive, I too was tested. I was kept under quarantine but by God's grace, my report turned out to be negative. I understand my family's worry.

D: There's a lot of anxiety among my family members, especially my wife and mother. I have been working for many years. But this happen first time that she told me that if you can skip going to work than skip it. As she is badly scared of it she thought that I may contract it from my duty. Then I tell her that this is my job and I have to do it.

D: when my 4 year old child comes running to hug me, I ward him off from afar, and he says; oh mommy after Covid I will hug you a million times.

D: this will require effort, but has to be done. If you say everything is fine, I am just going to be getting on with my life as if nothing will happen, then you are setting yourself up for infection.

Exhausted ENT doctors battle surging coronavirus cases. They are exhausted and facing staff shortages after nearly eight months of relentless work. Doctors are facing lots of stress, anxiety and vulnerability for contracting the infection. Many of them explains how much anxiety they feels and even mentions how a colleague got an anxiety attack in front of a patient. Many studies have suggested that healthcare workers who are in close contact with patients of this emerging infectious diseases like SARS, MERS, Ebola, and H1N1they will suffer from loneliness, anxiety, fear, fatigue, sleep disorders, and other physical and mental health problems [11-15]. Doctors are actually stressed out and they need time to step away and de-stress just like anyone else although that's not exactly possible during the pandemic.

D: It is very tiring mentally as well as physically. Before the pandemic, all the work in the hospital would be done without any hurdles. But now, the patient load, necessity for precautions, the mental engagement, the paperwork and physical work have increased manifold. And after donning the PPE, extra physical strength needed to do all this work.

D: If you find wearing a mask for an hour or two to be a task, than think about wearing a complete PPE kit for six hours while serving patients, it is actually nauseating, you can't feel anything, your hands will be numb in the gloves, you will be sweating profusely and you will be feeling tachycardia (i.e. your heart rate will be increased). The temperature of your body shoots up and you start feeling dizzy. The longer you spend wearing the kit, the longer all these reactions will last. But, you have to do it for the patients.

D: when I was taking blood samples, and the blood just splashed on my face shield and PPE. Naturally, I got very scared.

D: We usually don't get time to manage the stress.

D: The moment I went home, I take a bath and sleep. As after few hours, my next shift is to be started. To handle all these emotion and thought is actually tough at that time. So at home I just sleep, and don't waste my time to absorb all those emotions which I experience throughout the day.

D: Everyone is mentally exhausted doctor at the ICU. It requires continuous levels of attention and care.

D: We are exhausted by this, but the cases are exponentially rising, that's why we are doctors and we have to do this. We only have a handful of people we can rotate in.

D: It is very depressing. After my shift (once a week), I feel short of breath and have a sore throat for about two days. A friend of mine too experiences it. We don't know if it is psychological or it is really happening to us.

D: Obviously there is panic. Patients tend to get restless, but we can't lose our calm. The patients in the ICU are typically in bad shape. They have extreme difficulty in breathing, and most of them are above 45 years. Even though everything is in accordance with protocol, we still don't know what's going wrong sometimes.

$\mathrm{D}$ : The problem is that there is no literature available. Studies keep on changing, it's mostly trial and improvisation, at this point. The challenge is that there is no specific treatment. It's predominantly supportive care, and depending on a case-to-case basis, one can try medication or therapy.

The issues of uninterrupted supply of PPE remain the main reason to wear the suffocating kit for long duty hours and uneven timing of duty rotation increases the frustration among them. As duty hours are longer and very hectic.

D: our duty hour is of $6 \mathrm{~h}$ and even longer if you include donning and doffing time of PPE. We have to continue our duty with PPE without drinking water and going to washroom. Extreme dehydration and fatigue decreasing our efficiency.

D2: You are trying to do something, and nothing is working. Whenever I see intubated patient of Covid, the only thing come to my mind is, 'oh my God, the patient is going to die. And you know what is very disheartening.

$\mathrm{D}$ : our duty hour is of $6 \mathrm{~h}$ and even longer if you include donning and doffing time of PPE. We have to continue our duty with PPE without drinking water and going to 
washroom. Extreme dehydration and fatigue decreasing our efficiency.

D2: You are trying to do something, and nothing is working. Whenever I see intubated patient of Covid, the only thing come to my mind is, 'oh my God, the patient is going to die. And you know what is very disheartening.

D: We are working on alternate 12-h duties shifts for continuous 14 days then we get a 14-day quarantine break. Seven days we are spending in a hotel room provided to us, and the remaining seven at home.

D: I am scared to go home, because of the fear that I will infect my parents. As both are old and having comorbidities. So, I just can't risk being there with them. I rented a room outside where I am staying alone during the quarantine days, because I believes that being a doctor I not only have a responsibility to treat patients but also to protect the remaining citizens from infections. D: We suppose to maintain social distance when we are outside. So I completely avoid going out only in extreme emergency I stepped out. You can imagine how lonely and isolated we are. But for doctors like us, service comes first, even when you have to wear protective gear for six hours.

D: We maintain distance when outside so that if we have an infection, we don't give it to anybody else. So, I usually don't go near anyone when I am outside the hospital or room. I usually completely avoid going outside, until and unless it is absolutely necessary for me," she explains. Yes, that can be as lonely as you can imagine, but for doctors like her, service comes first, even when you have to wear protective gear for six hours.

D: We usually don't get time to manage the stress.

D: The moment I went home, I take a bath and sleep. As after few hours, my next shift is to be started. To handle all these emotion and thought is actually tough at that time. So at home I just sleep, and don't waste my time to absorb all those emotions which I experience throughout the day.

Doctors are more vulnerable to this new virus because of more exposure to more number of patients. So they are administering to their patients is only devoted care, companionship to a degree and repurposed drugs that may or may not work depending on the prognosis of the patient. But they cannot guarantee them complete recovery.

D: In a way, as doctors, we feel as helpless as the patients because we don't know everything about COVID-19 and we don't have a treatment or cure that'll work $100 \%$.We're just trying to make our patients feel comfortable, treat them as much as we can, and assure them that we're here for them.
As patients are lying in the wards without their family or loved one, they need mental support as well or just simple words that 'you'll be okay'. Sometimes, they die without getting a last glimpse of a loved one. It's very emotional when the last words they'll ever say are to us, and not their families. As a human being very impossible to come out of it.

D: There was one old patient, about 80 years. I was taking vitals, that was deranging when I saw that he couldn't even open his eyes and his breath was so laboured, I recalled my grandfather final days. I held his hands and asked, "Baba, are you okay?" But he didn't respond. He just held my hand tight and wouldn't leave it. I don't know how long I was there, just holding his hand and stroking his head to give whatever little comfort I could. "I didn't move away until one of my colleagues came and took me away. Once I left the room, I couldn't hold it in any longer. I just hugged my colleague and cried.

D: During the pandemic, time both compresses and elongates. The day passes by in a frenetic whirlwind, and the toll on the body is harsh, yet a 12-h shift can feel like a 24.

D: Everyone is mentally exhausted doctor at the ICU. It requires continuous levels of attention and care.

D: We are exhausted by this, but the cases are exponentially rising, that's why we are doctors and we have to do this. We only have a handful of people we can rotate in.

D: It is very depressing. After my shift (once a week), I feel short of breath and have a sore throat for about two days. A friend of mine too experiences it. We don't know if it is psychological or it is really happening to us.

D: Obviously there is panic. Patients tend to get restless, but we can't lose our calm. The patients in the ICU are typically in bad shape. They have extreme difficulty in breathing, and most of them are above 45 years. Even though everything is in accordance with protocol, we still don't know what's going wrong sometimes.

D: The problem is that there is no literature available. Studies keep on changing, it's mostly trial and improvisation, at this point. The challenge is that there is no specific treatment. It's predominantly supportive care, and depending on a case-to-case basis, one can try medication or therapy.

D: I felt like I was going to war or a radioactive zone. After wearing the gear, you can't breathe but just survive in it.

D: The PPE provided to us is not of top quality. We were not given adequate training initially and learned a lot of things including wearing PPE, through YouTube instead. 
D: There is a lot of stigma attached to the virus, whether it's patients or health care workers. People are getting apprehensive. A lot of our colleagues suffer when they get back home for the people living around them feel that it's they who'll spread the virus.

D: I got admitted in hospital and stayed there for 17 days. It is difficult to spend time all alone during the period without family and friends by the sides. At that time mine stood by me.

D: During the pandemic, time both compresses and elongates. The day passes by in a frenetic whirlwind, and the toll on the body is harsh, yet a 12-h shift can feel like a 24 .

There is need of hours for adequate rest (both physical and psychological) as well as clear communication, personal sense of control and support from higher authorities and society. As we all know low support and high workload are established risk factors that affect the mental health. Alden et al. also reported the same that low support and more workload are the main risk factors effecting the mental health problems among other occupational groups in times of this pandemic [16].

D: There is tremendous pressure on current health systems, so it is the need of the hour to improve the healthcare system for better organization, delivery and distribution of health care. For learning prospective the pandemic has paved the way for a more collaborative, digitally enabled and flexible workplace.

D: we can learn about better, smarter ways of working that seem to be improving the job satisfaction of younger doctors as well as the quality of care for their patients. One such example is the accelerated adoption of virtual care; online consultations to tele-ICUs, telehealth has become important tool for coping with this pandemic.

\section{Conclusion}

We are going through unprecedented times which brought unexpected consequences hitherto. As this pandemic shown us how healthcare is need not just tweaking, but radical change. While caring for extremely sick and dying patients infected with the coronavirus which is not their speciality, our ENT doctors have risked-and even losttheir lives to answer the call of a lifetime during this pandemic. Our daily practices have been changed. So it is necessary to reformulate a plan to guarantee the best management for the patients as well as doctors specially ENT doctors. Resilient feature of the present review is its comprehension and depth to assess the mental health of ENT doctors under the covid-19 pandemic. This qualitative designs helped us to contextualize and assess mental health with self-reported preferences. Many innovative ideas has been shared like limiting exposure opportunities, setting up independent teams of senior residents to maintain safe clinical practice while preserving trainees' safety. On-line journal clubs/ webinars and online consultations were the other most important activity during pandemic; moreover, online lectures and for patients telehealth had the advantage of sharing knowledge among different centres.

\section{Compliance with Ethical Standards}

Conflict of interests No conflict of interests or funding has been disclosed for this article.

\section{References}

1. Jie R, Xilin Y, Zhen X, Lei W, Kun Y, Yonggang K et al (2020) Prevention of nosocomial COVID-19 infections in otorhinolaryngology-head and neck surgery. WJOHNS. https://doi.org/10.1016/j.wjorl.2020.06.003

2. Zou L, Ruan F, Huang M et al (2020) SARS-CoV-2 viral load in upper respiratory specimens of infected patients. N Engl J Med 382:1177-1179

3. Drosten C, Gunther S, Preiser W et al (2003) Identification of a novel coronavirus in patients with severe acute respiratory syndrome. N Engl J Med 348:1967-1976

4. Wang D, Hu B, Hu C et al (2020) Clinical characteristics of 138 patients with 2019 novel coronavirus-infected pneumonia in Wuhan, China. JAMA. https://doi.org/10.1001/jama.2020.1585

5. US Centers for Disease Control and Prevention. Interim Infection and Control Recommendations for Patients with Suspected or Confirmed Coronavirus Disease (COVID-19) in Healthcare Settings

6. Interpretation of pneumonia diagnosis and treatment scheme for novel coronavirus infection (trial version 6) [EB/OL] (2020) http://www.nhc.gov.cn/xcs/zhengcwj/202002/ 8334a8326dd94d329df351d7da8aefc2.shtml. Accessed 2 February 2020

7. Givi B, Schiff BA, Chinn SB et al (2020) Safety recommendations for evaluation and surgery of the head and neck during the COVID-19 pandemic. JAMA Otolaryngol Head Neck Surg. https://doi.org/10.1001/jamaoto.2020.0780

8. Buzatto GP, Tamashiro E, Proenca-Modena JL, Saturno TH, Prates MC, Gagliardi TB et al (2017) The pathogens profile in children with otitis media with effusion and adenoid hypertrophy. PLoS ONE 12:e0171049

9. Guidance for undertaking otological procedures during COVID19 pandemic [Internet]. https://www.entuk.org/guidance-undertaking-otologicalprocedures-during-covid-19-pandenmic. cited 1.4.20

10. Heikkinen T, Thint M, Chonmaitree T (1999) Prevalence of various respiratory viruses in the middle ear during acute otitis media. N Engl J Med 340:260-264

11. Chung BPM, Wong TKS, Suen ESB, Chung JWY (2005) SARS: caring for patients in Hong Kong. J ClinNurs 14:510-517

12. Kim Y (2018) Nurses' experiences of care for patients with Middle East respiratory syndrome-coronavirus in South Korea. Am J Infect Control 46:781-787

13. Khalid I, Khalid TJ, Qabajah MR, Barnard AG, Qushmaq IA (2016) Healthcare workers emotions, perceived stressors and 
coping strategies during MERS-CoV outbreak. Clin Med Res 1303:1-22

14. Liu C, Wang H, Zhou L et al (2019) Sources and symptoms of stress among nurses in the first Chinese anti-Ebola medical team during the Sierra Leone aid mission: a qualitative study. Int $\mathbf{J}$ Nurs Sci 6:187-191

15. Honey M, Wang WY (2013) New Zealand nurses perceptions of caring for patients with influenza A (H1N1). Nurs Crit Care 18:63-69
16. Alden LE, Matthews LR, Wagner SL et al (2020) Systematic literature review of psychological interventions for first responders. Work Stress. https://doi.org/10.1080/02678373.2020. 1758833

Publisher's Note Springer Nature remains neutral with regard to jurisdictional claims in published maps and institutional affiliations. 